\title{
CRIMSONpublishers
}

http://www.crimsonpublishers.com

Opinion

Med Surg 0phthal Res

ISSN 2578-0360

\section{Electromagnetic Waves Propagate Well in Insulating Biomaterials}

\author{
Shengyong $\mathrm{Xu}^{*}$ and Jingjing $\mathrm{Xu}$ \\ Department of Electronics, Peking University, P. R. China
}

*Corresponding author: Shengyong Xu, Key Laboratory for the Physics \& Chemistry of Nanodevices, and Department of Electronics, Peking University, Beijing, 100871, P. R. China

Submission: 眥 October 03, 2017; Published: 監 October 30, 2017

\section{Opinion}

Manmade electrical circuits and chips are made of solid materials cataloged as transistors, resistors, capacitors, inductors, interconnects, etc., where electrons are the charge carriers running inside conductors and semiconductors, characterized with terms such as electric currents and voltages, and insulators separate conducting paths. In natural biosystems, electrical communications are also widely observed. For instance, when a brain is working, one may record complicated electrical voltage signals from an array of electrodes attached to the brain. However, natural biosystems are mainly made of soft materials such as protein, phosphorus membrane and water-based fluid. In these systems, ions of $\mathrm{Na}+, \mathrm{Ca} 2+, \mathrm{K}+$, etc. are utilized as the charge carriers instead of free electrons. How does the electrical information propagate in biosystems? Whether it propagates via ion currents [1-3], by electromechanical solitons [4], or by soliton-like electromagnetic pulsed waves [5]? This remains an open argument to date.

There is a huge difference between an electric current and an electromagnetic (EM) wave. The former describes the flux of moving charges, but the latter describes the change of electric and magnetic fields. Gama rays, X-rays, ultraviolet lights, visible lights, infrared lights, T-Hz ways, microwaves, radio waves, etc., they are all EM waves with different wavelengths. Their behaviors all follow Maxwell equations and propagate in vacuum or insulators at a speed of the order of $108 \mathrm{~m} / \mathrm{s}$. One of the unique properties of EM waves is that they all travel perfectly in vacuum without losing energy over a very long distance, therefore one sees the lights of stars and galaxies millions and billions of light years away from the Earth. EM waves also prefer to travel in dielectrics. The vacuum has an infinite resistance, while dielectrics are perfect insulators. EM waves reflect almost hundred percent when meeting a metallic surface, and when meeting an electrolyte, or a conducting fluid with ions, EM waves are partially reflected back. This partial reflection makes the sandwiched electrolyte-membrane-electrolyte structures e.g. axons and microtubules good soft material waveguides [5].

On sharp contrast, under a voltage where the voltage keeps its sign in time, an electric current can only propagate in a conductor or semiconductor, but not in insulators or vacuum, no matter the charge carriers are free electrons or ions. However, under an AC voltage where the voltage periodically changes its sign in time, a weak electric current can pass across an insulator, such as the dielectrics in a capacitor. In biosystems, no ac voltage exists, therefore electric currents only transport in the conducting paths filled with ionic fluids.

Now it is clear that in natural biosystems if the electrical information is carried by an electric current, it takes the path of an ionic fluid, e.g., the core of an axon. If the electrical information is carried by an EM wave, it takes the path of an insulator, e.g. the membrane of an axon. In some cases, e.g. in a brain or a heart, bioelectrical information may be carried by both currents and EM waves.

We stated that a membrane plays three main roles for electrical communication in a biosystem [6]. It serves as the isolating dielectrics for the universal electrical power supply in live biosystems, an electrolyte-membrane-electrolyte capacitor which is charged by naturally formed transmembrane gradient of ion concentration in aqueous solution. It serves as the matrix for transmembrane $\mathrm{Na}+\mathrm{K}+$ and $\mathrm{Ca} 2+$ protein channels, which are capable of generating soliton-like pulsed EM waves by transient transmembrane ionic currents once triggered open. And, it serves as the framework of soft-material waveguides for transmission of the soliton-like pulsed EM waves generated by ion channels.

Various weak photon emissions, referred as "biophotons", have been detected since 1920's in bacteria, plants, animal cells, even in the nerve systems of human beings [7-9]. Researchers observed that visible lights generated by biochemical reactions could propagate efficiently in membrane-based microtubules acted as optical waveguides [10]. When passing through a chain of cyanobacteria in a suspension, lights were surprisingly found focused better as they were traveling in an optic waveguide [11]. Our recent experiments of soft material waveguides, where the lipid membrane was replaced with plastic material, showed a much higher transmission efficiency for EM waves as compared to the cases when they are 
propagating in pure liquid or air [12]. For transmission of pulsed EM waves, the cutoff period is around $10 \mu \mathrm{s}$, corresponding to a frequency of $100 \mathrm{kHz}$, and the transmission efficiency increases with the thickness of the dielectrics. Our simulation results also confirmed that the concept of "soft material waveguide" [5] is valid at the scales of centimeter and millimeter. As Maxwell equations are supposed to stay valid from the macroscales to the nanoscale, we believe the $2-3 \mathrm{~nm}$ thick membrane of unmyelinated axons, normal cells, and tubes in biosystems serves well as the transmission path for pulsed EM waves.

This scenario is different from the widely accepted Hodgkin and Huxley $(\mathrm{H} \& \mathrm{H})$ theory [1-3]. $\mathrm{H}$ \& $\mathrm{H}$ theory describes the transmission behavior of action potentials with a circuit model based on equivalent resistors (ion channels and the cytosol of the axon) and capacitor (lipid membrane). For example, the socalled "Saltatory Conductance" of action potentials on myelinated axons is explained by the mechanism employing local circuit ionic currents that flow between nodes of Ranvier. The myelin sheath, which is made of tens of lipid bilayers and has a thickness up to a few microns, is only treated as the insulating shell that changes the capacitance in the conducting circuit [13]. Here the "screen effect" of the electro magnetics might have been overlooked. Indeed, according to Maxwell equations, rearrangement of the locations of ions (charges) at one node of Ranvier cannot create a stead transversal electric field (thus a dc voltage) along the axon for driving an ion current from one node to the next [6]. In another case, $\mathrm{H} \& \mathrm{H}$ theory faces difficulty in explaining the experimental observation for cross-over of two action potentials along one axon $[4,14]$.

Similar to the model of soliton-like pulsed EM waves, the electromechanical soliton model describes soliton-like mechanical waves as the information carrier. It assumes that when protein channels are triggered open a node of Ranvier, the transmembrane ion flows generate a local heat. This local heat results in a local phase change in the lipid membrane, and it propagates along the axon as a soliton-like mechanical dilatational wave, thus triggering ion channels at the next node [4]. The model was used to explain the refractory period, reversible channel phenomena from thermodynamics of the membrane without employing molecular features of the membrane components. Simulations showed that the minimum velocity of the solitons is close to the propagation velocity in unilamellar vesicles [4]. However, local temperature increment due to a transmembrane ion flux may not be sufficient to create a phase change in the lipid membrane, because the large volume fluid surrounding the channel could remarkably dissipate heat. For instance, in a recent experiment, local temperature increment in cultured adhesive cells was detected directly with micro-thermal sensors underneath the cells, showing only a weak increment of 30-300mK [15]. Energy-wise a soliton-like EM pulse (e.g., a photon) transmits within the lipid membrane with a much higher efficiency than a soliton-like mechanical wave does.

By analyzing the "simultaneous phenomena" observed in unicellular organisms, plants and animals, we recognized that natural biosystems had developed soft material waveguide networks and extensively utilized soliton-like EM pulses for fast and synchronized activities. It is indeed a "Wireless Communication" mechanism [16]. For example, when a paramecium touches a subject on its way, it can reverse from swimming forward to swimming backward within only $30 \mathrm{~ms}$. This is done by changing the beating direction of about 5000 cilia simultaneously [17]. In trapping action of a flytrap, a plant, an EM pulse generated at the sensory receptor of one leaf can result in quick reaction for millions of cells located at the junction part of the two trapping leaves within 1s [18]. As an extreme case, an electric eel can discharge up to 400 times per second [19], and it can simultaneously discharge 109-11 ion channels within 0.1-0.5ms for one killing performance, corresponding to a signal propagation speed of $1000 \mathrm{~m} / \mathrm{s}$ or more. The model of transmission of soliton-like EM pulses in coupled membrane-based waveguides explains consistently these unique phenomena $[5,16]$.

In short, being lack of transversal electric voltage across nodes of Ranvier or between neighboring cells, natural biosystems may not have used electric currents for transmission of electrical information, but used soliton-like pulsed EM waves instead. EM waves travel well in various membrane-based networks among cells and cell organelles [20]. Taking this point of view, axons are specially developed paths for optimized transmission efficiency for EM pulses. This may shed some light for understanding how a brain works because of the complicated electrical communication behaviors among billions of neurons in a brain may base on the same physical principle.

\section{References}

1. Hodgkin AL, Huxley AF (1945) Resting and action potentials in single nerve fibres. J Physiol 104(2): 176-195.

2. Hodgkin AL, Huxley AF (1952) A Quantitative Description of Membrane Current and its Application to Conduction and Excitation in Nerve. J Physiol 117(4): 500-544.

3. Bear MF, Connors BW, Paradiso MA (2007) Neuroscience: Exploring the brain ( $3^{\text {rd }}$ Edn). Lippincott Williams \& Wilkins Inc, USA.

4. Heimburg T, Jackson AD (2005) On soliton propagation in biomembranes and nerves. Proceedings of the National Academy of Sciences of the United States of America 102(28): 9790-9795.

5. Xue JW, Xu SY (2012) Natural electromagnetic waveguide structures based on myelin sheath in the neural system. Quantitative Biology.

6. Xu SY, Xu JJ, Yang F (2016) The Roles of Membrane for Electrical Communication in a Biosystem. Neuroscience and Biomedical Engineering 4(4): 230-236.

7. Fritzalbert $\mathrm{P}$, Beloussov LV (2003) Integrative biophysics. Biophotonics Journals.

8. Popp FA, ChangJJ, Gu Q Ho MW (1994) Nonsubstantial Biocommunication in Terms of Dickers Theory.

9. Gurwitsch A (1926) Das Problem der Zellteilung physiologisch betrachtet. Protoplasma 1(1): 473-475.

10. Thar R, Kühl M (2004) Propagation of electromagnetic radiation in mitochondria? J Theor Biol 230(2): 261-270.

11. Anna Bezryadina TH, Rekha Gautam, Benjamin Wetzel, Graham Siggins, Andrew Kalmbach JL, et al. (2017) Nonlinear Self-Action of Light through 
Biological Suspensions. Physical Review Letters 119(5): 058101.

12. Li G, Pang TW, Sun WQ, Luo L, Pang JF, et al. (2014) Transmission of Electromagnetic Waves in Soft material Waveguides. Applied Mechanics \& Materials 513-517: 3625-3629.

13. Raven P, Singer S, Mason K, Johnson G, Losos J (2014) Biology (10 ${ }^{\text {th }}$ Edn).

14. Gonzalez-Perez A, Budvytyte R, Mosgaard LD, Nissen S, Heimburg T (2014) Penetration of Action Potentials During Collision in the Median and Lateral Giant Axons of Invertebrates. Physical Review X 4(3): 031047.

15. Yang F, Li G, Yang J, Wang Z, Han D, et al. (2017) Measurement of local temperature increments induced by cultured HepG2 cells with microthermocouples in a thermally stabilized system. Scientific Reports 7(1): 1721.
16. Xu JJ, Yang F, Han DH, Xu SY (2017) Wireless Communication in Biosystems. Biological Physics.

17. Naitoh Y, Eckert R (1969) Ionic Mechanisms Controlling Behavioral Responses of Paramecium to Mechanical Stimulation. Science 164(3882): 963-965.

18. Autho (2006) Electrical signals in long-distance communication in plants. Springer Berlin Heidelberg.

19. Catania K (2014) The shocking predatory strike of the electric eel. Science 346(6214): 1231-1234.

20. (2005) 125 Questions: What don't we know? Science 309(5734): 558558. 